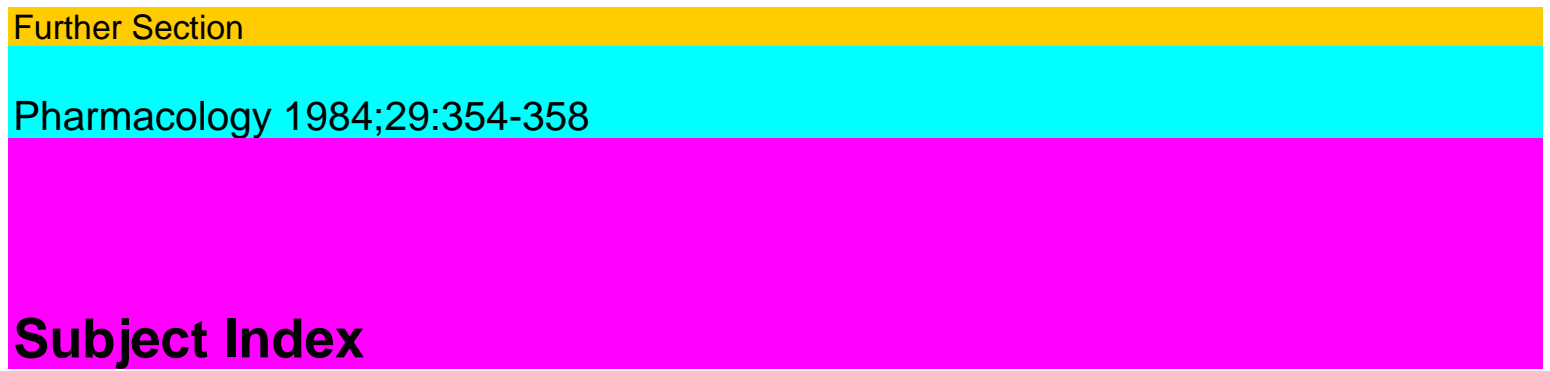

Acetaminophen 149 Acetylcholinesterase 40 Acetylstrophanthidin 224 Acid secretion 320

Aconitine 193 ACTH 269 Adrenal gland 94 Adrenalectomy 269, 276 B-Adrenergic receptor

247 Adrenergic responsiveness 173 Adrenoceptor antagonists 233

- $\quad$ blocking action 204

ß-Adrenoceptor blocking action 241

- regulation 210

Adventitia 17,20

Affinity 198

N-Allylnormetazocine 325 Alprenolol 247

Amino acids $185 \gamma$-Aminobutyric acid 24 Aminoglycosides 292 Aminopyrine 128 14CAminopyrine accumulation

320 Aminopyrine, N-demethylation

128 Amobarbital 128 Amphetamine 269 Analgesia 269 -, opioid-mediated 278 -, stressmediated 275 Analgesic activity 193

-, determination 194 Aneurysm 1 Angiotensin 61

II 63

Antagonist, calcium 165,282

-, calmodulin 75

-, $\mathrm{CaM}, 75$

-, cholinergic 329

Antianginal agents 198

Antibiotics 292 Anticancer drugs 110 Antihypertensive agents 241 Anti-inflammatory drugs 193 Antipyrine 99, 110, 117, 128 Aorta 255

Aortic smooth muscle 173 Arachidonic acid 25 Aromatic amino acid decarboxylase 185

hydrocarbons 121 Arotinolol 204 Arterial blood pressure 63

spasm 1

strips 25

wall 255 Artery, dog basilar 26

-, isolated coronary 241 -, pulmonary 165 -, renal 17 Aryl amines 193

- $\quad$ hydrocarbon hydroxylase 121 ,

143

Aspirin 24

Assay, prolyl hydroxylase 255

Atenolol 210

Atropine 329

Autoradiography 247

AV block 224

Bacterial endotoxin 70 
lipopolysaccharide 70 Basilar artery 24 Benzene-isoamyl alcohol 158 Benzo(a)pyrene hydroxylase 142

metabolites 142 Benzodiazepines 158 Bethanechol 329 Bicuculline 24

Bile duct 301

- $\quad$ - cannulation 51

Biliary drainage 132

excretion 301 Binding, radioreceptors 18

studies 200 Bioavailability 305 Biogenic amines 185

-, extraction and separation

186

-, neuronal uptake 269 Biological response 149

systems 181 Biosynthesis, collagen 255 Blood 1,71, 173,210,215,255,

282 -, vasospastic compounds 5

- $\quad$ vessels 70, 165

Bowel 47

Bradykinin 70

Brain 85, 94

Bronchodilatation 247

Bronchospasm 282, 286

-, antigen-induced 282

-, histamine-induced 282 Butaclamol 75

Calcium antagonists 1, 9, 198, 282

blockers 282

channel 165

- blocker 264,282

entry blockade 198

ion flux 224 Calmodulin 75 Cancer, human 143 Canine portal vein 233 Cannabidiol 343

Cannabinoids 343 Cannabinol 343 Cardiac arrhythmias 264

output 215 Cardioprotection 61 Cardiopulmonary functions 283 Cardioselective drugs 210

Cardioselectivity 210

Subject Index

355

Carrageenin 193 ß-Casomorphin 325 Catecholamines 1,173,247,269 Cathaedulis 269

Cathartic agents 47 Cathinone 269 Ca uptake 165 Cell culture 344 Cells, pancreatic acinar 185 Cerebral vasospasm 1 Chloramphenicol 117 Chlorophenylalanine 269 Chlorothiazide 255 Cholestyramine 128 Cholinesterase 40 Cinanserin 19 Circadian rhythms 149 Clearance 110 -, antipyrine 110 CNS depression 128 Cocaine 337 Collagen 255

- $\quad$ synthesis, determination 256

Compound 48/80 70

Conjugation 301

Constriction 1

Contraction 24, 165,233

-, mechanisms 3

Contracture 242 
Convulsions 85

-, electroshock-induced 86

Corn oil 121

Coronary artery $61,204,241$

Corticosteroid therapy 117

Covariance 181

Creatine kinase 65

- $\quad$ - activity 61

Cysteine 301

Cytochrome P-450 121, 142

DADLE 320, 325 Dark 149

Decarboxylation 185 Dermorphin 56 Desoxycorticosterone 173 Development 181

Dexamethasone 94, 95, 269 Diazepam 158 Diazoxide 282 Dietary thiamin 142

Diet, thiamin-deficient 142 Diethylstilbestrol 121 Digitalis 224

Digoxin 224 toxicity 224

Dihydroalprenolol 247

Dihydroergosine 215

Dihydroergotamine 336

Dihydromorphine 325

Dilazep 198

DOC A 255

Dog 24,204,224,241

coronary artery 204 L-Dopa, decarboxylation 185 Dopamine 17

receptors 17 Droperidol 19

Drug disposition 117,312

intake 305

interactions 224

oxidation 158 Drugs, elimination 99 Dynorphin 325

Electron microscopy 292 Enalapril 61

Endoplasmic reticulum 143 ß-Endorphin 325 Endothelial cells 17

damage 1

fenestrae 292 Enteric-coated pellets 305 Environmental chemicals 143 Enzymes 181

-, acetylcholinesterase 40

- $\quad$ inhibitor 61

Epinephrine 247

Equilibrium dialysis 312,314

Ergosinine 215

Ergot alkaloids 215

- derivatives 215

Erythromycin 305

-, absorption and bioavailability

305 -, antibiotic assay 306 -, serum concentrations 305 Estradiol 121

- benzoate 121

Estrogens 121

Ethanol 94 
- $\quad$ triglycerides 94

Ethyl acetate 100

Ethylketazocine 325

Ethylsuccinate 305

Etiocholanolone 70

Evipan ${ }^{\circledR} 100$

Exocrine pancreas 185

Fatty liver 94 Feeding studies 142 Femoral veins 336 Fluorescence 75 Flurothyl 85 Food consumption 149 Fundic strips, guinea pig stomach 330

GABA 24

Gas chromatography 158

liquid chromatography 264 Gastric fundus 329

secretion 329

smooth muscle 329 Gastrointestinal transit 47 Gentamicin 292 Germinal cells 343

Glomerular capillary 292

permeability 292

ultrastructure 292 Glomeruli, ultrastructural

evaluation 293 Glucuronic acid 301 Glutathione 149,301 Gonadal hormones 121,124

Guanethidine 255 Guinea pig heart 166

- $\quad$ - ileum $31,32,56$

Haloperidol 215 Heart 204

atrium contraction 165

rate 198,210,215 Hemodialysis 312 Hemoglobin 1 Hemorrhage 1 Hepatectomy 110 Hepatic

function 110

malfunction 99

- $\quad$ microsomal function

110

356

Subject Index

Hepatic function (cont.)

- $\quad$ mixed function oxidases 110 ,

128

Hepatotoxicity 301 Heterogeneity 75 Hexobarbital 99 High-clearance compound 99

Histamine 233, 282, 320 Histoautoradiography 17 Human femoral artery 336

- vein 336

mononuclear leukocytes 210

subjects 128

- $\quad$ volunteers 305

Hydrocortisone 117

15-Hydroperoxyarachidonic acid Hydroxy metabolites 158 4-Hydroxyantipyrine 996 -

Hydroxydopamine 233 3'-Hydroxyhexobarbital 99 3-Hydroxymethylantipyrine 99 5-

Hydroxy tryptophan 185 Hypertension 210,241,255 Hypertensive patients 210

Hypertriglyceridemia 94 Hypotension 70 Hypothermia 128 -, antipyrine 129

Ibuprofen 85 
Imidazobenzodiazepines 158 Indole derivatives 193 Indomethacin 24 Interaction 198 Intestinal transit 47 Intima 17,20 Intoxication 71 Inulin 70 Ischemia 61 Isolated coronary artery 241

dog coronary artery 204

enriched parietal cells 320

guinea pig gastric fundus 329,330

left atrium 166

nerve 31

papillary muscle 167

pulmonary artery 166

- $\quad$ spermatogenic cells 343

Isoproterenol 173,247

3'-Ketohexobarbital 99 Kinin 70, 72 Kyotorphin (Arg-Tyr) 325

Lethality, acetaminophen 149 Leu5-enkephalin 320 Leukocytes 70, 210 -, ß2-adrenoceptors

210 Light 149

Lipopolysaccharide 70 Liver 94, 181

damage 110

lysosomes 61 Low-clearance compound 99 Lung 247 Lymphocytes 210

Man 301

Marihuana 343

Mass spectroscopy 158

Meclofenamate 85

Media 20

Mediators, vasospasm 8

Mefenamate 85

Mercapturate 301

Mesenteric artery 255

Metabolic pathways 99

Metabolism 99

Metabolites, cumulative excretion

in urine 104 Methoxamine 198 3-Methylcholanthrene 142 Methyldopa 255 Methysergide 336 - dimaleate 25

Metoclopramide 19

Metoprolol 247

Mice 149

Microsomal enzymes 117 Midazolam 158

Mixed function oxidases 121, 128,

142 Model substrates 99 Morbidity 1 Morphine 47, 269 -, antidiarrheal effects 47 Mortality 1

Muramyl dipeptide 31 Muscarinic receptors 40, 329 Myocardial amino-nitrogen 61

ischemia 61

oxygen 61 Myoepithelioid cells 292

Naja venom 70 Naloxone 56, 269, 320 Natriuresis 17 Neonatal imprinting 121

Nephrotoxicity 292 -, aminoglycosides 292 Netilmicin 292 Neutrophil leukocytes 70 
Nifedipine 165,282 Nipradilol 241 Nitrendipine binding 165 Nitroglycerin 241 Nitrogenphosphorus detection

264 Nomifensine 269 Nonesterified fatty acids 312 Nonopiate analgesia 269 Nonsteroidal anti-inflammatory

agents 193,312 Noradrenaline 233, 336

- $\quad$ release 233

Norantipyrine 99

Norepinephrine 173,204,205,

241,247 Norverapamil 264

Opiate analgesia 269

Opiates 47

Opioid peptides 56

Opioids 269, 320

-, effect on gastric acid secretion

320 Ovariectomy 121 Oxazolone 193

Palmitic acids 312 Pancreas 40

acinar cells 185 Papillary muscles 61

- action potential 165 Paracetamol 301

cysteine 302

glucuronide 302

mercapturate 302

sulfate 302 Paralysis 128

Subject Index

357

Parietal cells 320 Patients, hypertensive 210 -, uremic 312 Pellets 305 Pentetrazol 85

Pentobarbital 129,204

- $\quad$ sleep times 129

Pentolinium tartrate 25

Peripheral resistance 215

Peripubertal period 121

Permeability 292

Pharmacokinetic parameters,

erythromycin 309

- $\quad$-, verapamil 267

Pharmacokinetics $128,158,181$

-, erythromycin 305

-, pentobarbital and antipyrine

130 -, verapamil 264 Phenobarbital 100, 142 Phentolamine 19,233

- $\quad$ mesylate 25

Phenylepinephrine 216

n-Phenyl-/-naphthylamine 75

Phosphodiesterase 75

Picrotoxin 24

Pindolol 247 Pirenzepine 329 Pithed rat 198 Pizotifen 336 Plasma 94, 158,264

glycoside concentrations 224 


\section{protein 312}

- binding 312 Polycation effects 292 Portocaval shunt 99 Portal vein 233 Postnatal

development 40 Postsynaptic adrenoceptors 198 Potassium 241

chloride 173 Prazosin 233 Prednisolone 117

acetate 95 Prenylamine 75, 165 Pressure-rate index 61 Proline, hydroxylation 255 14C-

Proline 255 Prolonged analgesia 269 Prolyl hydroxylase 255

Propranolol 204,241,247 Prostaglandins 1,7,24,85

- $\quad$ synthetase 85

Protocollagen 255

Pseudocholinesterase 40

Puberty 121

Pulmonary artery contraction 165 Pyrilamine maleate 25

Rabbit 17, 70, 117 Radioligand binding assays 247 Radioreceptor binding studies 17 Rat 85, 94 -, adrenalectomized 94

DOCA-NaCl-treated 173

hypertensive 255

neonatal female 121

newborn female 122

spontaneously hypertensive

215 Receptor autoradiography 247 -, $\beta$-adrenergic 247

- $\quad$ density 210

-, dopamine 17

-, 5-hydroxytryptamine 336 -, muscarinic 40, 329 -, non- $\mu$-opioid 269 Relaxation 17,24,241

Release, endogenous

catecholamines 233 Renal artery 17

dialysis 316

excretion 301

failure 312 Reserpine 255, 269 Reserpinization 32 Ricinoleic acid 47 RNA synthesis 343

Rupture 1

Salbutamol 285 Salmonella 70

- $\quad$ minesota 71

$+\mathrm{H}-$ Secretion 320

Seizure 85

-, flurothyl-induced 86 Serotonin 1,31,269 Sex difference 121

- $\quad$ hormones 122

Sinus rhythm 224

Smooth muscle 31, 329

- cells 17 Sodium chloride 173

nitrite 173 Soterenol 247 Soybean lipoxygenase 25 Sperm 343 Spermatocytes 343

Spermatogenesis 343 Spiroperidol 17 Stearate 305 Stomach strip 31 Stress 94

analgesia $269 \mathrm{~K}-$ Strophantin 165 Structure-activity relationships

56 Subarachnoid hemorrhage 1 Sulfate 301 Sulindac $\mathbb{R} 85$

Sympathomimetic agents 247 Systolic blood pressure 173, 256

Tachycardia 224 Tachyphylaxis 32, 233 Tail-flick 56

- test 269 Temporal effects 47 Terbutaline 247 Testosterone 121

enanthate 121 
propionate 121 Tetraethylammonium 94, $95 \Delta$ 9-Tetrahydrocannabinol 343 Therapy, cerebral vasospasm 8 Thiamin-deficient diet 142 Thoracotomy 204

Timolol 247

Tobramycin 292

Tolerance 269

Tolmetin 312

-, plasma protein binding 316

Total peripheral resistance 215

Toxicity, glycosides 224

- $\quad$ studies 195

Tracheal pressure 286

Trifluoperazine 75

Triglyceride 94

Tubular damage 292

Tyramine 233

358

Subject Index

Ulcerogenic activity 194 Uremia 312 Uridine 343 Urinary excretion 99

Vagus nerve 47 Variables 181 Vasdeferens 31, 56 Vascular reactivity 173 - smooth muscle 17

Vasoactivity 61 Vasoconstriction 1,24,198 Vasodilatation 17,241 Vasodilators 9 Vasospasm, cerebral 1 Vein, canine portal 233 Ventricular arrhythmia 224 - ectopy 224 Verapamil

$165,224,264,282$

Vessels, cerebral blood 1 Vinylbital 100

Weaning 40

White blood cells 71

Yohimbine 215,233

Zoxazolamine 128 